\title{
Collagen Induction Therapy With Dermaroller
}

\author{
* Deepali $\mathbf{B}^{1}$
}

\section{ABSTRACT:}

Dermaroller is relatively a new modality for the treatment of acne scars, stretch marks, wrinkles, facial rejuvenation inducing collagen induction by the use of microneedling. Two versions are available: (1) the standard dermaroller version and (2) miniature versions. The principle of using dermaroller modality is to provide collagen induction therapy. Dermaroller is more effective in rolling and boxcar type of scars. It has been shown that rolling with a dermaroller (192 needles, $200 \mu \mathrm{m}$ length and $70 \mu \mathrm{m}$ diameter) over an area for 15 times will result in approximately 250 holes/ $\mathrm{cm} 2$. Microneedling leads to the release of growth factors form new collagen and elastin in the papillary dermis. In addition, new capillaries are formed. The neovascularisation and neocollagenesis lead to reduction of scars. The procedure is aptly called "percutaneous collagen induction therapy" and is also used in the treatment of photoageing. Dermaroller is an extremely effective therapy for post acne scars, even trauma scars. Best results are seen in rolling and box type scars, and even papular scars respond to it.

Key words: Collagen induction therapy, dermaroller, microneedling, scars.

\section{Introduction}

The development of microneedling occurred in 1994. Electroridopuncture (ERP) for skin aging and acne scars was practiced in cosmetic dematology since 1995 when Orentreich and Orentreich described subcision or dermal needling for scars ${ }^{1}$. Camirand and Doucet in 1997 evidenced described needle dermabrasion using a "tattoo pistol" to treat scars $^{2}$. Recently in 2006 Fernandes developed amore cost-effective management by introducing percutaneous collagen induction therapy with the dermaroller ${ }^{3}$.

Dermaroller is relatively a new modality for the treatment of acne scars, stretch marks, wrinkles, facial rejuvenation inducing collagen induction by the use of microneedling. It is simple, safe and cost effective form of management which can be combined with few more techniques like punch elevation, excision, subscision, etc on the same or different sittings depending on the individual patient requirements. Since dermaroller is suitable for all types and tones of skin and has indications and results similar to fractional and resurfacing lasers with the lack of issues of ablation and patient downtime, therefore it is definitely proving to be a new advanced treatment option.

\section{Dermaroller modality for collagen induction therapy:}

The 'dermaroller' is a rolling device model used for collagen induction therapy by dermatosugeons. Two versions are available:

(1) The standard dermaroller version is a drumshaped cylindrical roller equipped with 192 fine stainless steel microneedles arranged in eight rows each measuring $0.5-3.0 \mathrm{~mm}$ in length, $0.1-0.25 \mathrm{~mm}$ in diameter, width $20 \mathrm{~mm}$. According to the applied pressure they penetrate the skin upto papillary dermis.
The instrument is presterilized by gamma irradiation technique and should be cleaned with betadine and normal saline before using. Of course, needle length is critical but, needle diameter is more important as we are seeking to maximize the dermal injury without creating a new scar.

(2) Miniature versions called derma-stamps are also available which are used for localized scars, eg. varicella scars and their needles are $2 \mathrm{~mm}$ in length with a diameter of only $0.12 \mathrm{~mm}$.

\section{Principle and functional mechanism Dermaroller}

The principle of using dermaroller modality is to provide collagen induction therapy. This is done by causing a minute injury to the dermis with use of microneedles. This results in starting of the wound healing cascade and hence utilizing the body self-healing mechanisms. Skin reacts to the intrusion of micro-needles like any other skin penetrating object. In this new device the needles are so fine and thin that tissue damage is unlikely. The skin integrity actually stays intact and only the body's own production of collagen is stimulated. However, this "nerve-stimulus", transported by electrical signals, triggers the cascade of the healing process releasing growth signals to undifferentiated cells and phase 1 inflammation starts immediately after the injury and later these signals in return stimulate the proliferation of new cells, e.g. fibroblasts to transform into collagen- and elastin fibres by 5 th day -8 weeks. The task of fibroblasts is, to migrate to the point of intrusion for wound closure and this new fibre formation thickens the skin and fills former atrophic scars and this body reaction is called neo-collagenesis. In addition, the inner cells that coat our vessels (endothelial cells), and in particular these of our capillaries, are also stimulated to proliferate thus inducing neo-angiogenesis. The natural tissue remodeling will continue from 8 weeks up to nearly 1 year.

1. 'Dr. Deepali Bhardwaj, M.D (Dermatology), M.Phil,

Consultant \& Director, Dr. Deepali Skin Clinic, New Delhi, India

* for correspondence

Email : drdeepali1@yahoo.com

Website: www.drdeepali.com 
It has been shown that rolling with a dermaroller (192 needles, $200 \mu \mathrm{m}$ length and $70 \mu \mathrm{m}$ diameter) over an area for 15 times will result in approximately 250 holes $/ \mathrm{cm} 2$. Microneedling leads to the release of growth factors which stimulate the formation of new collagen (natural collagen) and elastin in the papillary dermis. In addition, new capillaries are formed-this neovascularisation and neocollagenesis following treatment leads to reduction of scars $4,5,6$. The procedure is therefore aptly called "percutaneous collagen induction therapy" and has also been used in the treatment of photoageing. The figure below illustrates diagrammatically the functional mechanism of collagen induction therapy by Dermaroller technique $7,8,9$
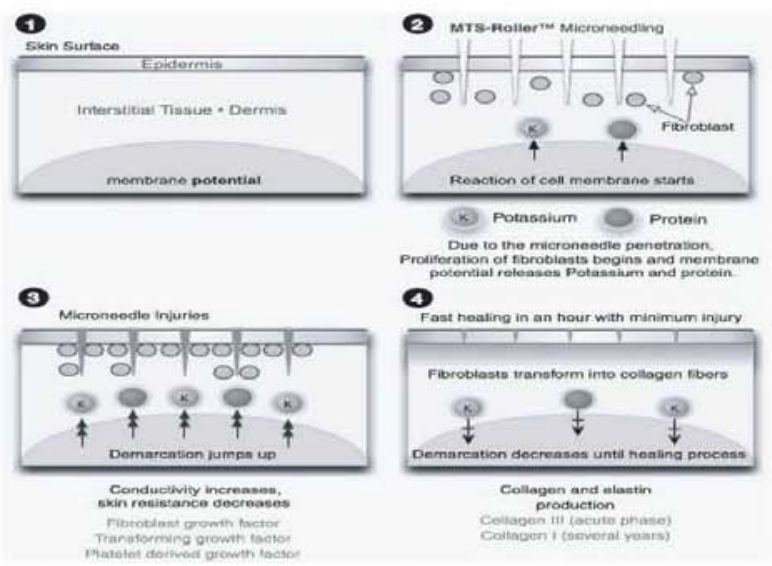

Fig.1 Diagrammatic illustration of the functional mechanism of collagen induction therapy by Dermaroller technique.

Indications and contraindication of collagen induction therapy by Dermaroller technique

\section{Indications}

1. Scars: Atrophic scar, hypertrophic scar, achromic scar of any etiology, post acne, burns, chicken pox etc

2.. Skin ageing: Facial wrinkle and laxity, sagging and folding

3. Large pores

\section{Absolute contraindications}

1. Bleeding/coagulating disorders

2. Extreme keloidal tendency.

3. Presence of skin cancer, warts, solar keratosis.

4. On chemotherapy, radiotherapy and high doses of corticosteroids

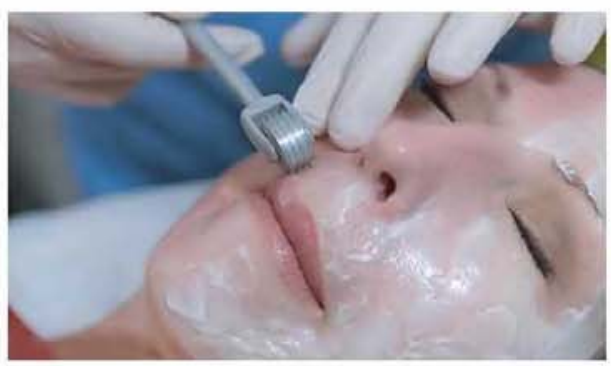

\section{Efficacy of Dermaroller:}

The diagram presented below shows that dermaroller is more effective in rolling and boxcar type of scars ${ }^{10}$.

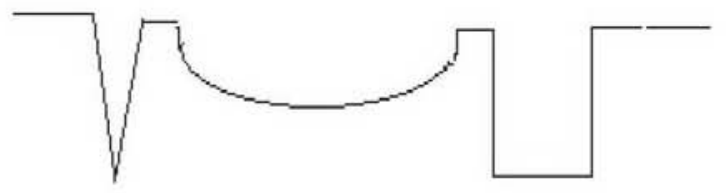

\begin{tabular}{|c|c|c|}
\hline ICEPICK SCARS & ROLLING & BOXCAR \\
\hline${ }^{*}$ Narrow $(<2 \mathrm{~mm})$ & ${ }^{*}$ Wide (>4-5mm) & * $1.5-4 \mathrm{~mm}$ in diameter. \\
\hline $\begin{array}{l}\text { *Extend vertically to } \\
\text { the deep dermis or } \\
\text { subcut tissue }\end{array}$ & $\begin{array}{l}\text { - Dermal tethering of } \\
\text { of relatively normal } \\
\text { appearing skin }\end{array}$ & $\begin{array}{l}\text { * Shallow } \\
\text { * Round to oval depression } \\
\text { 'Sharply demarcated vertical edges }\end{array}$ \\
\hline
\end{tabular}

\section{Procedure of collagen induction therapy by Dermarol- ler technique}

It is a simple OPD procedure. The area to be treated is anesthetized with topical anesthesia for 45 minutes to one hour. After preparation of the area, rolling is done 5 times each in horizontal, vertical, and oblique directions. Petechiae or pin-point bleeding is the end point and after treatment. The area is wetted with saline pads and topically purascarbol, taurinox or sialorgamine can be applied. The entire procedure lasts for 15 to 20 minutes, depending on the extent of the area to be treated. A minimum of four weeks is recommended between two treatments as it takes long time for new natural collagen to form. Three to four treatments may be needed for moderate acne scars.

\section{Post-therapy care and management procedure care} Microneedling is well tolerated by patients but erythema may be seen after treatment, lasting for 2-3 days. Photoprotection is must during treatment and also local antibiotic creams may be prescribed. The patients can go back to work the same day also. Apart from erythema, no other side effects have been reported.

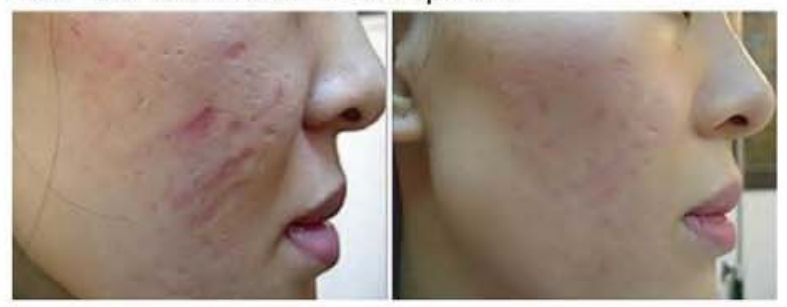

\section{Practical Tips}

1. Combination treatment with subscision, punch elevation, fractional laser gives excellent results.

2. Adding topically arbutin, silourgamine, taurinox gives better results specially in facial rejuvenation

3. If facial redness does not disappear in 2 days, adding anti-inflammatory is an option

4. Explaining the patient complete procedure in detail before starting the series of session is a must along with expectations. 


\section{Conclusion:}

Dermaroller is an extremely effective therapy for post acne scars, even trauma scars. Best results are seen in rolling and box type scars ,even papular scars respond it, but ice pick scars show no great improvement. It is also, an extremely cost effective tool and hence, very popular in practice for a Dermatologist.

\section{References}

1. Orentreich DS, Orentreich N. Subcutaneous incisionless (subcision) surgery for the correction of depressed scars and wrinkles. Dermatol Surg 1995; 21:6543-9.

2. Camirand A, Doucet $J$. Needle dermabrasion. Aesthet Plast Surg 1997; 21:48-51.

3. Fernandes $D$. Minimally invasive percutaneous collagen induction. Oral Maxillofac Surg. Clin North Am 2006; 17:51-63.

4. Aust MC, Fernandes D, Kolokythas $P$, Kaplan HM, Vogt PM. Percutaneous collagen induction therapy: An alternative treatment for scars, wrinkles and skin laxity. Plast Reconstr Surg 2008; 21:1421-9.

5. Fernandes $D$, Signorini $M$. Combating photoaging with percutaneous collagen induction. Clin Dermatol 2008; 26:192-9.

6. Aust MC, Reimers K, Repenning C, Stahl F, jo ahn S, Guggenheim $M$, et al. Percutaneous collagen induction: Minimally invasive skin rejuvenation without risk of hyperpigmentation-fact or fiction. Plast Reconstr Surg 2008; 122: 1553-63.

7. Goodman GJ, Baron JA. Postacne scarring: A qualitative global scarring grading system. Dermatol SurgeJ Am Acad Dermatol 2001; 45:109-17.

8. Jacob CL, Dover JS, Kaminer MS. Acne scarring: A classification system and review of treatment options. $J$ Am Acad Dermatol-2001; 45: 109-117.

9. Doddaballapur S. Microneedling with dermaroller. $J$ Cutan Aesthet Surg-2009; 2:110-1.

10. Aust MC, Fernandes D, Kolokythas P, Kaplan HM, Vogt $P M$. Percutaneous collagen induction therapy: An altemative treatment for scars, wrinkles and skin laxity. Plast Reconstr Surg 2008; 21:1421-9. 